\title{
La familia Conidae en el mar peruano
}

\section{The family Conidae from Peruvian Sea}

\section{Carlos Paredes ${ }^{1,2}$, Franz Cardoso ${ }^{1,2}$, Katherine Altamirano, Paul Baltazar y Leonardo Romero ${ }^{1}$}

1 Laboratorio de Biología y Sistemática de Invertebrados Marinos, Facultad de Ciencias Biológicas, Universidad Nacional Mayor de . San Maros, Apdo. 11-0058, Lima, cparedesq@unmsm.edu.pe 2 Departamento de Malacología, Museo de Historia Natural, Universidad Nacional Mayor de San Marcos, Apdo. 14-0434, Lima 14, Perú.

Trabajo presentado a la XVIII Reunión Científica del Instituto de Investigaciones en Ciencias Biológicas Antonio Raimondi, "200 años del nacimiento de Charles Darwin y el 150 aniversario de la publicación de On the Origin of Species by Means of Natural Selection". Del 19 al 21 de agosto de 2009 .

\section{Resumen}

En el presente trabajo se describe y proporciona información sobre las especies del género Conus (Neogastropoda: Conidae) mantenidas en el Laboratorio de Biología y Sistemática de Invertebrados Marinos de la Facultad de Ciencias Biológicas (LaBSIM) y en el Departamento de Malacología y Carcinología del Museo de Historia Natural de la Universidad Nacional Mayor de San Marcos (MUSM). Se estudió material colectado en 9 localidades del Departamento de Tumbes, y 16 del Departamento de Piura. La colecta intensiva se llevó a cabo en abril del año 2007 y fue esporádica en los años posteriores. De las 16 especies reportas para Perú hasta antes de este estudio, se cuenta con material correspondiente a 11 de ellas: Conus (Conus) gladiator Broderip, 1833; Conus (Asprella) arcuatus Broderip \& Sowerby, 1829; Conus (Cylinder) lucidus Wood, 1828; Conus (Leptoconus) poormani Berry, 1968; Conus (L.) recurvus Broderip, 1833; Conus (Pyruconus) ferguson Sowerby, 1873; Conus (P.) patricius Hinds, 1843; Conus (Ximeniconus) mahogani Reeve, 1843; Conus (X.) perplexus Sowerby, 1857; Conus (X.) tornatus Sowerby II, 1833; y Conus (X.) ximenes Gray, 1839 . No se tiene material de Conus (C.) princeps Linnaeus, 1758; Conus (C.) tiaratus Sowerby, 1833; Conus (Chelyconus) purpurascens Sowerby, 1833; Conus (Leptoconus) regularis Sowerby, 1833; y Conus (L.) virgatus Reeve, 1849. Conus kohni MacLean \& Nybakken, 1979 y Conus xanthicus Dall, 1910 son reportadas por primera vez, con lo cual aumenta a 18 el número de especies registradas para el mar tropical del Perú.

Palabras claves: Mollusca, Gastropoda, Caenogastropoda, Conoidea, nuevos registros, Perú.

\section{Abstract}

This paper describes and provides information on the species of the genus Conus (Neogastropoda:Conidae) preserved in the Laboratorio de Biología y Sistemática de Invertebrados Marinos de la Facultad de Ciencias Biológicas (LaBSIM) y el Museo de Historia Natural de la Universidad Nacional de San Marcos (MUSM). The material was collected at the Department of Tumbes (9 locations) and Piura (16 locations). Intesive collect was realized in April 2007 and occasional in posterior years. Of the 16 reported species it is counted with material corresponding to 11 of them: Conus (Conus) gladiator Broderip, 1833; Conus (Asprella) arcuatus Broderip \& Sowerby, 1829; Conus (Cylinder) lucidus Wood, 1828; Conus (Leptoconus) poormani Berry, 1968; Conus (L.) recurvus Broderip, 1833; Conus (Pyruconus) fergusoni ;Sowerby, 1873; Conus (P.) patricius Hinds, 1843; Conus (Ximeniconus) mahogani Reeve,1843; Conus (X.) perplexus Sowerby, 1857; Conus (X.) tornatus Sowerby, 1833 and Conus (X.) ximenes Gray, 1839. There are no material of Conus (C.) princeps Linnaeus, 1758; Conus (C.) tiaratus Sowerby, 1833; Conus (Chelyconus) purpurascens Sowerby, 1833; Conus (Leptoconus) regularis Sowerby, 1833 and Conus (L.) virgatus Reeve, 1849. Conus kohni MacLean \& Nybakken, 1979 and Conus xanthicus Dall, 1910, are reported for the first time, wich increases to 18 the number of species registered for the tropical sea of Peru.

Keywords: Mollusca, Gastropoda, Caenogastropoda, Conoidea, new records, Perú.

\section{Introducción}

La Familia Conidae ( Prosobranchia: Neogastropoda) está constituida actualmente por 546 taxa específicos y subespecíficos del género Conus, que se distribuyen en la zona tropical de los océanos del mundo, la mayoría en el Indopacífico y el Pacífico Occidental (Röckel et al. 1995, Tucker 2004). La forma y la coloración de una especie de Conus puede variar de una localidad a otra, pero manteniendo algunos caracteres relativamente uniformes (Keen 1971, Keen \& Coan 1975), lo cual ha ocasionado mucha confusión y conflictos taxonómicos (Díaz et al. 2005), así como dificultades en la caracterización de especies nuevas, e innumerables casos de sinonimia. Tomlin (1937) incluyó en su catálogo más de 2700 nombres para las especies vivientes y fósiles; ahora hay más de 2000 nombres para la designación de las especies vivientes de Conus y otro tanto para las fósiles (Tucker 2004). López (2001) señala que Conus es el género de invertebrados marinos con el mayor número de especies vivientes, lo que es consecuencia de su gran éxito evolutivo, basado principalmente en el sofisticado método de alimentación depredadora que les permite cazar la presa, paralizarla y en muchos casos, engullirla casi instantáneamente utilizando la rádula toxoglosa provista de dientes marginales que tienen forma de arpón e inyectan veneno.
No obstante el gran número de especies de la familia, los intentos para establecer una clasificación genérica han fracasado debido a la relativa uniformidad de la forma de las conchas, y hay muchas clasificaciones propuestas, faltando información anatómica que posibilite la revisión del género (Rosenberg 1992).

La tendencia actual es mantener al género como una unidad indivisible (Walls 1979, Khon 1990), considerando que las subdivisiones propuestas se basan en caracteres externos sin valor filogenético. Los estudios de biología molecular contribuirán a la solución de este problema (Díaz et al. 2005).

Los cónidos como integrantes del bentos marino, habitan diversos tipos de sustrato, incluyendo fondos rocosos, arenosos, fangosos, arrecifes de coral, fondos cubiertos de vegetación, etc., desde la zona intermareal hasta los $1000 \mathrm{~m}$ de profundidad (Dance \& Cosel 1977), alimentándose en la noche de moluscos, gusanos y peces (Keen 1971).

Se ha sugerido una clasificación basada en el tipo de presa, considerándose especies vermívoras (poliquetos, equiuridos, y hemicordados), las cuales son mayoría (unas 300), especies moluscívoras y piscívoras (unas 50); entre estas últimas están las que son peligrosas para el hombre como Conus geographus, del Indo 
Pacífico (Díaz et al. 2005). Los dientes radulares de cada uno de estos grupos presentan un morfología similar, lo cual pudo ser un punto de partida para la creación de otros géneros. Sin embargo Nybakken (1970), quien estudió la rádula de muchas especies, encontró posteriormente cambios ontogenéticos en los tipos de rádula, otorgándoles significado en sistemática y ecología (Nybakken 1988, 1990). Cabe aquí señalar que se debe tener mucho cuidado al coger con la mano ejemplares vivos de cónidos, por razones obvias.

En el Pacífico Oriental Tropical, dentro de los límites de la Provincia Panameña, se han registrado 29 especies de Conus (Keen 1971; Abbott 1974), y más recientemente 40 especies (Skoglund 2002). En el Perú se conocen 16 especies del género Conus para el mar tropical peruano (Peńa 1970, Alamo \& Valdivieso 1987, 1997, Rivadeneira \& Injoque 1990, Paredes et al. 1999, Mogollón \& Vargas 1999).

Actualmente hay mucho interés en el estudio de las conotoxinas presentes en el veneno de estos gasterópodos depredadores, el cual es inyectado cada vez por un único diente desechable y en forma de arpón de la rádula toxoglosa, que también sirve para la captura de la presa. Las conotoxinas son herramientas ideales en los estudios de neurofisiología debido a su elevada especificidad y posibilidad de sintetizarlas químicamente; y se considera que en el futuro próximo podrían ser de utilidad en el tratamiento de enfermedades del sistema nervioso (López 2001).

En el presente trabajo se describe y proporciona información sobre las especies del género Conus mantenidos en el Laboratorio de Biología y Sistemática de Invertebrados Marinos de la Facultad de Ciencias Biológicas (LaBSIM) y el Museo de Historia Natural de la Universidad Nacional Mayor de San Marcos (MUSM).

\section{Material y métodos}

El material analizado fue colectado mediante buceo autónomo con el apoyo de buzos artesanales en abril del año 2007, en los Departamentos de Tumbes (9 localidades) y Piura (16 localidades). Los especimenes de Conus kohni y C. xanthicus fueron colectados en septiembre 2008. Además se revisaron muestras colectadas antes del 2007 y en el año 2009, depositadas en las colecciones del Museo de Historia Natural de la Universidad Nacional Mayor de San Marcos (MUSM). Las muestras fueron fijadas en formol al 7\% neutralizado con bórax, y conservadas en alcohol etílico al 70\%. El ordenamiento supraespecífico está basado en Skoglund (2002), y la determinación taxonómica se realizó con la bibliografía especializada. Las fotografías fueron tomadas utilizando una cámara digital. El material fue depositado en el Laboratorio de Biología y Sistemática de Invertebrados Marinos de la Facultad de Ciencias Biológicas (LaBSIM) y el Museo de Historia Natural de la Universidad Nacional Mayor de San Marcos (MUSM).

\section{Resultados y discusión}

La Colección de Referencia del género Conus, en la Universidad Nacional Mayor de San Marcos está constituida por 14 especies, distribuidas en 48 lotes y 130 especímenes. Consideramos que el inventario del genero Conus en el Perú aún está incompleto debido a las dificultades para colectar en el sublitoral. Las especies que faltan en el Colección de Referencia son: Conus (Conus) princeps Linnaeus, 1758; Conus (Conus) tiaratus Sowerby, 1833; Conus (Chelyconus) purpuracens Sowerby, 1833; Conus (Leptoconus) regularis Sowerby, 1833; y Conus virgatus
Reeve, 1849. Conus xanthicus Dall, 1910 y Conus kohni MacLean \& Nybakken, 1979 se registran por primera vez para la fauna malacológica marina del Perú, con lo cual se incrementa a 18 el número de especies del género Conus que viven en nuestro mar tropical.

\section{Taxonomía}

\begin{tabular}{|c|c|}
\hline SUPERFAMILIA & CONOIDEA \\
\hline FAMILIA & CONIDAE \\
\hline GÉNERO & Conus Linnaeus, 1758 \\
\hline SUBGÉNERO & Conus, s. s. \\
\hline
\end{tabular}

\section{Conus (Conus) gladiator Broderip, 1833}

Figura 1

Conus gladiator Broderip, 1833. Proc. Zool. Soc. Lond., p. 54; Abbott, 1974:258.

Conus (Conus) gladiator, Peña, 1970; Keen, 1971: 661, fig. 1493; Alamo \& Valdivieso, 1987: 81; Paredes et al., 1999: 29; Skoglund, 2002: 161.

Concha con espira baja y amplia abertura, más abierta en el extremo anterior; vuelta corporal con coronaciones débiles en los hombros, las que se pueden reconocer en las vueltas de la espira, y líneas espirales débiles, más conspicuas en el extremo anterior; color del fondo blanco con dos bandas espirales de color pardo claro; periostraco fibroso en el sentido longitudinal y de color pardo oscuro; el interior de la abertura es blanco. Longitud 43,2 $\mathrm{mm}$; diámetro $27,4 \mathrm{~mm}$.

Distribución: Bahía Magdalena, Baja California, Golfo de California; Ecuador a Paita, Perú (Alamo \& Valdivieso 1987).

Hábitat: Mediolitoral rocoso (Alamo \& Valdivieso 1987).

Localidades: Tumbes (Zorritos, El Abejal).

Material examinado: 3 lotes, 5 ejemplares; fondo arenoso en el intermareal (Keen 1971).

\section{Subgénero Asprella Schaufuss, 1869}

\section{Conus (Asprella) arcuatus Broderip \& Sowerby, 1829}

\author{
Figura 2
}

Conus arcuatus Broderip \& Sowerby, 1829. Zool. Jour., 4: 379; Hendrickx \& Toledano, 1994: 59; Díaz et al., 2005: 77.

Conus (Asprella) arcuatus, Keen, 1971: 663, fig. 1496; Mogollón \& Vargas, 1999: 21-26; Skoglund, 2002: 161.

Conus (Lithoconus) arcuatus, Abbott, 1974: 258.

La altura de la espira es algo más de un tercio que la de la vuelta corporal, termina aguzada y su perfil es cóncavo; vuelta corporal con hombros carinados y el área bajo la sutura es ligeramente cóncava; abertura con canal posterior profundo, labio externo curvado sobre la abertura y reflejado dorsalmente en su extremo anterior; el amplio canal sifonal se levanta ligeramente hacia el lado derecho; la escultura presenta costillas espirales evidentes en toda la vuelta corporal; la coloración es blanca con tres zonas de manchas pardas, el interior de la abertura es blanco con manchas oscuras en el borde; el periostraco es fino y de color amarillo verdoso. Longitud 40,9 $\mathrm{mm}$; diámetro 18,7 mm.

Distribución: Bahía de San Carlos, Sonora, México; Esmeraldas, Ecuador, y hacia el sur hasta Punta Mal Pelo, Tumbes, Perú (Skoglund 2002). 

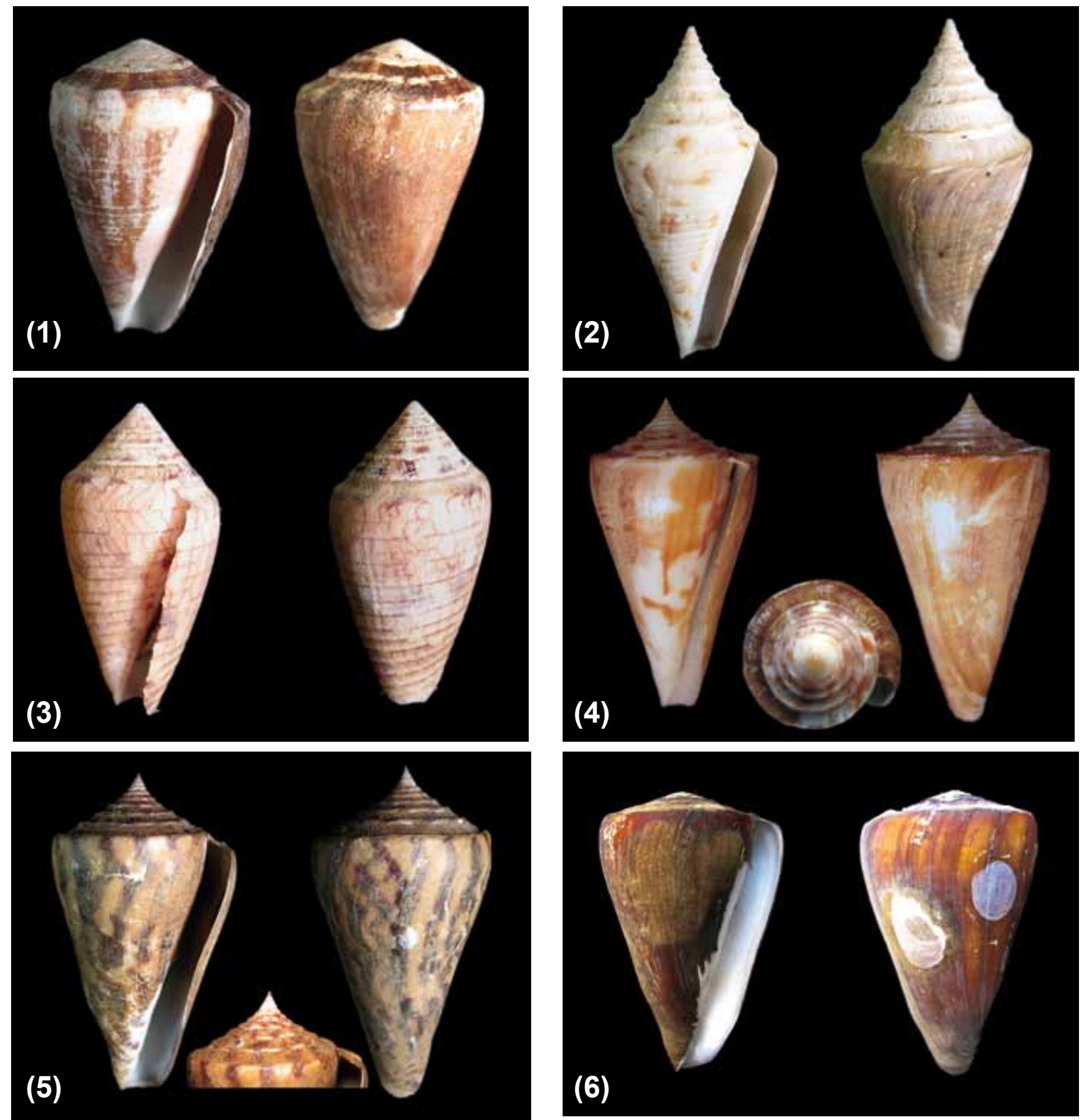

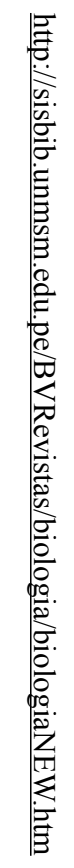
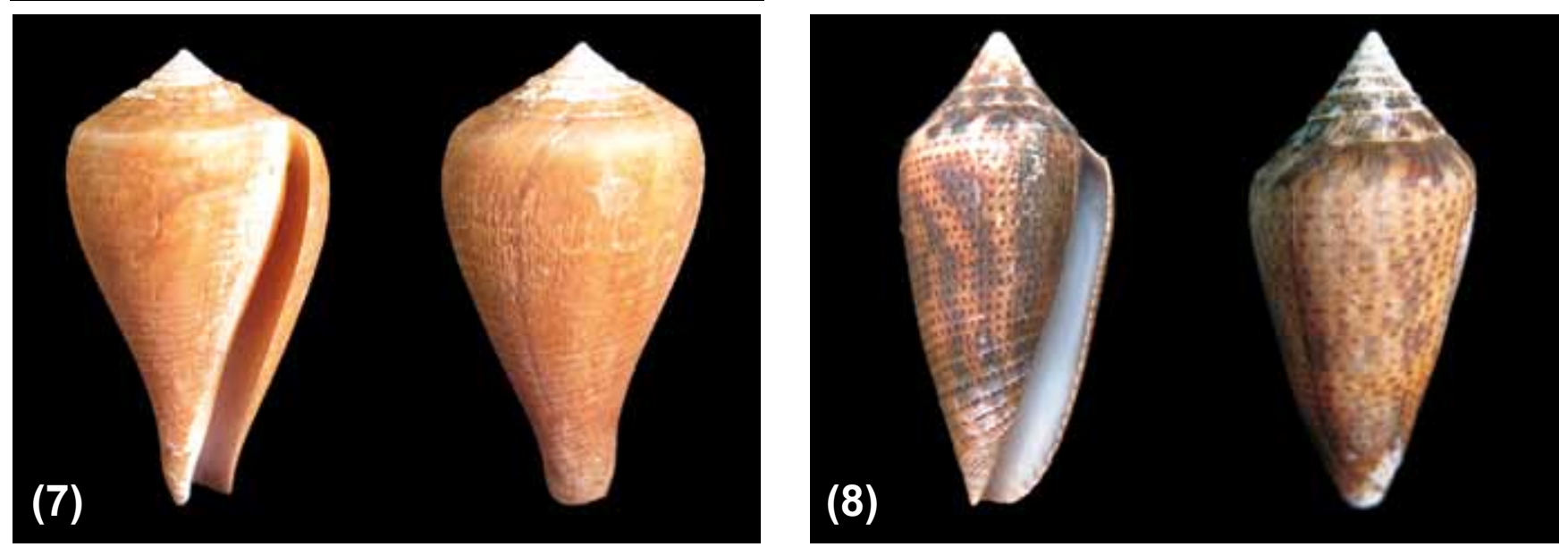

Figuras 1 - 8. (1) Conus (Conus) gladiator; (2) Conus (Asprella) arcuatus; (3) Conus (Cylinder) lucidus; (4) Conus (Leptoconus) poormani; (5) Conus (Leptoconus) regularis; (6) Conus (Pyruconus) fergusoni; (7) Conus (Pyruconus) patricius; (8) Conus (Ximeniconus) mahogani. 

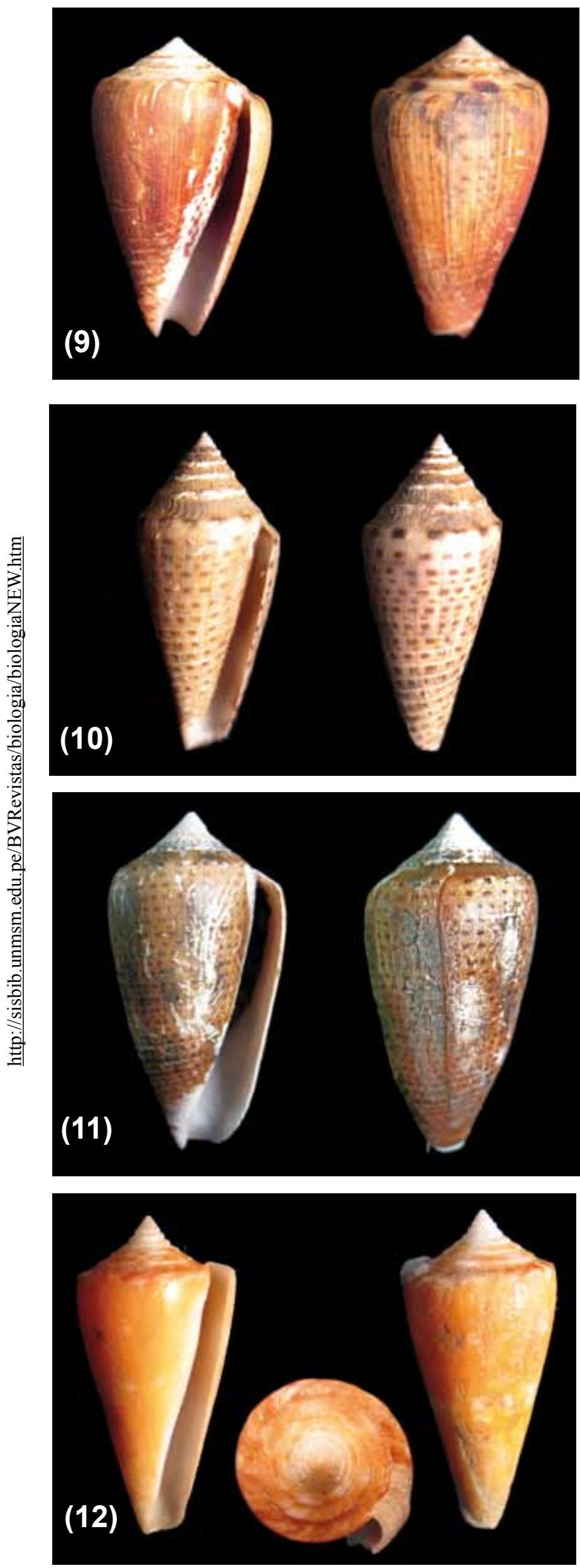

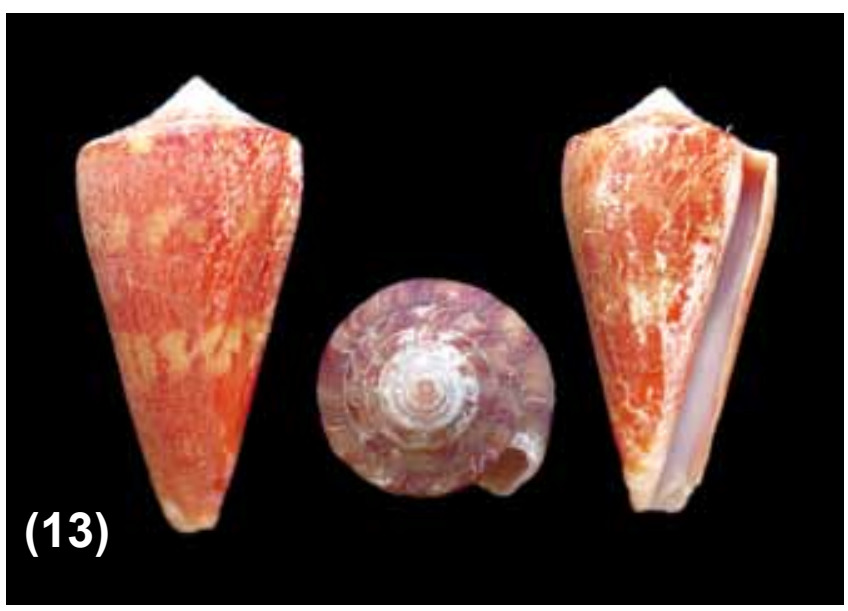

Figuras 9 - 13. (9) Conus (Ximeniconus) perplexus; (10) Conus (Ximeniconus) tornatus; (11) Conus (Ximeniconus) ximenes; (12) Conus kohni; (13) Conus xanthicus.

Hábitat: Sublitoral arenoso. Entre 61 y 109 m de profundidad (Hendrickx \& Toledano 1994).

Localidades: Tumbes (Quebrada Seca, Las Salinas), Piura (El Ñuro).

Material examinado: 3 lotes, 3 ejemplares; fondo arenofangoso, 24 - $30 \mathrm{~m}$ de profundidad.

\section{Subgénero Cylinder Montfort, 1810}

\section{Conus (Cylinder) lucidus Wood, 1828}

Figura 3

Conus lucidus Wood, 1828. Ind. Test. Suppl. p.8, pl. 3, fig. 4; Dall, 1909: 207; Abbott, 1974: 258, pl. 15, fig. 2825.

Conus (Cylindrus) lucidus, Keen, 1971: 664, fig. 1503; Alamo \& Valdivieso, 1987: 81, fig. 170.

Conus (Cylinder) lucidus, Paredes et al. 1999: 29; Skoglund, 2002: 162.

Espira alta de perfil recto, vuelta corporal con los hombros redondeados; abertura con seno posterior somero, más abierta en el canal anterior, borde del labio externo ligeramente crenulado; escultura consiste en costillas espirales bien separadas por interespacios ligeramente cóncavos, en casi la mitad anterior de la vuelta corporal, las que alcanzan el labio interno; color de la conchilla violeta pálido, con una malla de líneas espirales y axiales irregulares de color pardo oscuro, que recuerda los diseños de la antigua China; manchas grandes irregulares de color pardo oscuro forman una banda espiral en el centro de la vuelta corporal; la espira con manchas tipo flama y de color pardo oscuro; interior de la abertura de color violeta pálido brillante; el periostraco es delgado, sedoso y de color pardo claro. Longitud $32,0 \mathrm{~mm}$; diámetro $19,6 \mathrm{~mm}$.

Distribución: Bahía Magdalena, Baja California, Golfo de California; Ecuador a Punta Capones, Perú (Alamo \& Valdivieso 1987).

Hábitat: Sublitoral rocoso, 1 a $5 \mathrm{~m}$ de profundidad (Cantera et al. 1979; Cosel 1984; Blanco \& Cantera 1994).

Localidades: Piura (Peña Parada).

Material examinado: 2 lotes, 2 ejemplares fondo arenoso con rocas pequeñas, $13 \mathrm{~m}$ de profundidad. 


\section{Subgénero LePtoconus SWAINSON, 1840}

\section{Conus (Leptoconus) poormani Berry, 1968}

Figura 4

Conus (Leptoconus) poormani Berry, 1968. Notices of new eastern Pacific Mollusca-VII. Leaflets in Malacol., 1(25): 156; Keen, 1971: 665, fig. 1505; Abbott, 1974: 258, № 2812; Alamo y Valdivieso, 1987: 81; Skoglund, 2002: 163.

La espira es baja, aguzada y de perfil cóncavo, con una altura algo menor a la novena parte de la longitud total; la sutura es acanalada y la superficie bajo la misma es cóncava en las tres últimas vueltas y aún más en la vuelta corporal, la cual tiene los hombros carinados y los lados ligeramente cóncavos que terminan rectos en los hombros; la abertura presenta un canal posterior profundo, no es muy amplia y en su mitad anterior se abre terminando en un canal anterior muy amplio, debido a que el labio externo curvado se refleja hacia dorsalmente; color del fondo blanquecino brillante con estrías axiales y espirales, estas últimas más fuertes en el tercio anterior de la conchilla, hay gruesas manchas irregulares de color pardo claro amarillento, no muy densas en la vuelta corporal y en la espira, el color interior de la abertura es blanco opaco; periostraco delgado color pardo claro y piloso en los hombros y el area bajo la sutura de la vuelta corporal.Longitud $57,5 \mathrm{~mm}$; diámetro $28,4 \mathrm{~mm}$.

Distribución: Sonora, Mexico; Bahía Octavia, Colombia (Keen 1971) a Paita, Perú (Alamo \& Valdivieso 1987).

Hábitat: Infralitoral areno fangoso en profundidades de 55 a 70 m (Keen 1971) y hasta 165 m (Shasky 1997).

Localidades: Tumbes (Puerto Pizarro).

Material examinado: 1 lote, 1 ejemplar varado en el intermareal arenoso.

\section{Conus (Leptoconus) recurvus Broderip, 1833}

Figura 5

Conus recurvus Broderip \& Sowerby, 1833. Proc. Zool. Soc. Lond. p. 54; Dall, 1909: 207; Hendrickx \& Toledano, 1994: 59.

Conus (Leptoconus) recurvus, Keen, 1971: 665, fig. 1506; Alamo \& Valdivieso, 1987: 81, fig. 171; Paredes et al., 1999: 29; Skoglund, 2002: 163.

Conus (Lithoconus) recurvus, Abbott, 1974: 258, pl.15, fig. 2831.

La espira es ligeramente alta, de perfil cóncavo y aguzada, el área bajo la sutura es cóncavo; vuelta corporal con los lados algo cóncavos, los hombros fuertemente carinados hasta angulares; abertura amplia con in profundo canal posterior; labio externo oblicuo y flexionado por lo que la abertura es mas amplia en su tercio anterior, formando un canal muy abierto y algo flexionado dorsalmente; color del fondo blanco con bandas axiales irregulares pardo oscuras; interior de la abertura blanco violaceo; el periostraco delgado y liso, es de color pardo claro. Longitud 65,4 mm; diámetro $34,6 \mathrm{~mm}$.

Distribución: Bahía Magdalena, Baja California, Golfo de California; Colombia a Banco de Máncora, Perú (Alamo \& Valdivieso 1987).

Hábitat: Sublitoral areno-fangoso (Alamo \& Valdivieso 1987). Entre 0 y $91 \mathrm{~m}$ de profundidad (Hendrickx \& Toledano 1994).
Localidades: Tumbes (Puerto Pizarro, ensenada El Bravo, Quebrada Seca, Las Salinas), Piura (Máncora, La Bocana del Estero).

Material examinado: 6 lotes, 22 ejemplares; fondo fango arenoso, 24 - $30 \mathrm{~m}$ de profundidad. El ejemplar de Puerto Pizarro, varado.

\section{Subgénero Pyruconus Olsson, 1967}

\section{Conus (Pyruconus) fergusoni Sowerby, 1873}

\author{
Figura 6
}

Conus fergusoni Sowerby, 1873. Proc. Zool. Soc. Lond. p. 145, pl. 15, fig. 2; (Dall, 1909): 207; McLean \& Nybakken, 1979: 135-139, figs. 5-12, text. figs. 1-2; Kerstitch, 1989: 52, fig. 110; Hendrickx \& Toledano, 1994: 59

Conus (Lithoconus) fergusoni, Peña, 1970: 169; Keen, 1971: 667, fig. 1511; Abbott, 1974: 258, pl. 15, fig. 2829; Alamo \& Valdivieso, 1987: 82; Paredes et al., 1999:29.

Conus (Pyruconus) fergusoni, Skoglund, 2002: 164

La concha muy pesada tiene la espira baja comparado con los ejemplares de Panamá; vuelta corporal con hombros carinados, los espacios bajo la sutura ligeramente cóncavos; abertura amplia con seno posterior no muy profundo, el labio externo suavemente redondeado en su extremo posterior se abre en su tercio distal para formar un amplio canal anterior; coloración de la concha blanco, algunos ejemplares presentan dos bandas amarillentas rodeando la base de la vuelta corporal; periostraco aterciopelado muy adherente y de color pardo opaco; interior de la abertura blanco. Longitud 142,6 mm; diámetro 82,4 mm.

Distribución: Bahía Bartolomé, Baja California, Golfo de California; Santa Elena, Ecuador a Cherres, Perú (Alamo \& Valdivieso 1987).

Hábitat: Infralitoral areno fangoso (Alamo \& Valdivieso 1987). Entre 4,6 y 152,4 m de profundidad (Kerstitch 1989).

Localidades: Tumbes (Cancas), Piura (Los Órganos, Sechura, Parachique, Las Delicias, y frente a Lobos de Tierra).

Material examinado: 7 lotes, 14 ejemplares; fondo arenoso, 3 - $10 \mathrm{~m}$ de profundidad.

Observaciones: Esta especie es la que alcanza mayor tamaño entre los cónidos del Pacífico Oriental.

\section{Conus (Pyruconus) patricius Hinds, 1843}

Figura 7

Conus patricius Hinds, 1843. Ann. Mag. Nat. Hist., 11, p.256; Díaz et al., 2005:79.

Conus (Pyruconus) patricius, Keen, 1971: 667, fig. 1513; Abbott, 1974: 258, pl. 15, fig. 2813; Alamo \& Valdivieso, 1987: 82, fig. 173; Paredes et al., 1999: 29; Skoglund, 2002: 164.

La espira es baja y aguzada en los ejemplares pequeños y muy baja en los adultos; vuelta corporal en forma de pera con hombros subcarinados en los juveniles y carinados en los adultos; abertura amplia con seno posterior profundo en los adultos y poco desarrollado en los juveniles; el canal anterior se levanta ligeramente hacia el lado derecho; escultura con suaves costillas espirales en la vuelta corporal de los juveniles; conchilla de color blanquecino en los adultos y anaranjado pálido en los juveniles; el interior de la abertura es ligeramente azulado y el borde del 
labio externo es liso; periostraco de color pardo claro, con un patrón de arrugas entrelazadas, más notorio en los juveniles. Longitud 35,2 mm; diámetro $19,3 \mathrm{~mm}$, en un juvenil; 92,8 $\mathrm{mm}$ y $58,6 \mathrm{~mm}$ en un adulto.

Distribución: Nicaragua; Ecuador a banco de Máncora, Perú (Alamo \& Valdivieso 1987).

Hábitat: Sublitoral arenoso, fangoso, rocoso; 5 a $25 \mathrm{~m}$ de profundidad (Cosel 1984, Blanco \& Cantera 1994).

Localidades: Piura (Sechura, Plataforma Cabo Blanco, Lobos de Tierra).

Material examinado: 3 lotes, 4 ejemplares; fondo arenoso, $21 \mathrm{~m}$ de profundidad. Los dos ejemplares de Lobos de Tierra, varados.

\section{Subgénero Ximeniconus Emerson \& Old, 1962}

\section{Conus (Ximeniconus) mahogani Reeve, 1843}

Figura 8

Conus mahogani Reeve, 1843. Proc. Zool. Soc. Lond. p. 169; Keen, 1971; Díaz et al., 2005: 79.

Conus (Ximeniconus) mahogani, Keen, 1971: fig., 1517; Skoglund, 2002: 164.

La concha es más esbelta que en Conus ximenes, casi bicónica por la mayor altura de la espira, cuyo perfil varía entre recto y ligeramente cóncavo con una altura aproximada a un tercio de la longitud total de la conchilla; vuelta corporal con hombros redondeados; abertura ancha sin canal posterior y con amplio canal anterior; la escultura de la vuelta corporal se inicia como en Conus ximenes, algo bajo la mitad de la vuelta, y el número de surcos o mas bien canales, es similar, más anchos cerca del extremo anterior, donde los surcos están muy juntos; la espira presenta manchas irregulares de color pardo oscuro en cada vuelta, y un patrón similar de hileras de manchas rectangulares y cuadradas en la vuelta corporal, el tamaño de estas motas o puntos varía según lo ejemplares pudiendo ser desde regularmente espaciados hasta muy densos cuando las manchas son pequeñas; también se presentan, por lo general, manchas irregulares axiales de color pardo oscuro en la vuelta corporal y una hilera de puntos se ubica típicamente bajo los hombros; el interior de la abertura es blanco azulado y el color de fondo de la conchilla es blanquecino. Longitud 49,2 $\mathrm{mm}$; diámetro $22,7 \mathrm{~mm}$.

Distribución: Golfo de California a Panamá (Keen 1971) y las islas Galápagos, Ecuador (Finet 1991), hasta Máncora, Perú (Peña 1970, como Conus ximenes).

Hábitat: En el intermareal y sublitoral arenoso. Hasta $17 \mathrm{~m}$ de profundidad (Hutsell 1993).

Localidades: Tumbes (Zarumilla), Piura (Sechura, Parachique, Vichayo).

Material examinado: 3 lotes, 20 ejemplares; fondo arenoso, $10 \mathrm{~m}$ de profundidad. El ejemplar de Zarumilla, varado.

Observaciones: Según Tucker (2007) algunos tienen dudas sobre la identidad específica de ésta especie y Conus $(X$.) ximenes, pero se ratifica en su posición de que son dos especies relacionadas pero fáciles de diferenciar, no solamente por la morfología y coloración de la conchilla, sinó también porque $C$. ximenes carece de opérculo y $C$. mahogani si lo tiene. Además señala que hay diferencias estadísticas significativas en los parámetros de la conchilla, luego de medir 38 ejemplares de C. mahogani y 35 de C. ximenes, coincidiendo con los resultados de Chaney (1987).

Nosotros hemos diferenciado las dos especies solamente por la forma y coloración de la conchilla.

\section{Conus (Ximeniconus) perplexus Sowerby, 1857}

\author{
Figura 9
}

Conus perplexus Sowerby, 1857. Thes. Conch., vol. 3, p. 20, pl. 200 [Conus pl. 14], fig. 324; Abbott, 1974: 58, pl. 15, fig. 2820; Díaz et al., 2005: 79.

Conus (Ximeniconus) perplexus, Keen, 1971: 669, fig. 1515; Alamo \& Valdivieso, 1987: 82; Paredes et al., 1999: 29; Rivadeneira \& Arias, 1999: 88-92; Skoglund, 2002: 164.165.

La espira es baja con los espacios bajo la sutura suavemente cóncavos; vuelta corporal con los hombros subcarinados; abertura con seno posterior profundo y amplio canal anterior excavado dorsalmente hacia la izquierda, el labio externo es delgado, liso y transluce en su borde las manchas de la concha; vuelta corporal lisa, pero en el tercio anterior presenta costillas espirales anchas separadas por surcos, las cuales alcanzan el labio interno; color de la concha blanco violáceo con numerosas hileras espirales de manchas rectangulares y cuadradas de color pardo oscuro, grandes manchas irregulares del mismo color se localizan bajo los hombros y también en las vueltas de la espira; interior de la abertura violeta oscuro o claro; periostraco delgado, liso y de color pardo claro. Longitud 28,5 mm; diámetro 16,6 mm.

Distribución: Bahía Magdalena, Baja California, Golfo de California; Golfo de Guayaquil e islas Galápagos, Ecuador (Finet 1985; Cruz 1992), hasta El Rubio, Tumbes, Perú (Alamo \& Valdivieso 1987).

Hábitat: Infralitoral en fondo de arena gruesa, 36 a $73 \mathrm{~m}$ (Shasky 1997).

Localidades: Tumbes (Punta Mero, Punta Sal, Las Salinas), Piura (Bocana del Estero, Máncora, Cerro del Encanto).

Material examinado: 12 lotes, 23 ejemplares; fondo arenoso $\mathrm{y}$ areno fangoso, $11-30 \mathrm{~m}$ de profundidad .

Observaciones: Según Kameya et al. (1991), esta especie puede encontrarse formando parte de la fauna acompañante de los langostinos litorales del Perú. Cruz (1992) lo encontró en el Golfo de Guayaquil (48 ejemplares en 5 estaciones) en sedimento de arena fina entre 10 y $15 \mathrm{~m}$ de profundidad.

\section{Conus (Ximeniconus) tornatus Sowerby II, 1833, ex Broderip MS.}

\section{Figura 10}

Conus tornatus Sowerby, 1833. The Conch. Illust. pt. 29, fig. 25; Dall, 1909:207; Abbott, 1974: 258; Hendrickx \& Toledano, 1994: 59; Rivadeneira \& Injoque, 1990: 35-36, fig. 1.

Conus (Ximeniconus) tornatus, Keen, 1971: 669, fig. 1516; Rivadeneira \& Arias, 1999: 88; Skoglund, 2002:165.

Concha relativamente pequeña y frágil. La espira aguzada y de perfil recto alcanza una altura de, aproximadamente, un tercio de la longitud total; los espacios bajo la sutura son cóncavos; vuelta corporal con hombros carinados, rasgo que se puede apreciar 
en las vueltas de la espira; la abertura es estrecha y el borde del labio externo es delgado; seno posterior somero y canal anterior ligeramente levantado hacia la derecha: escultura consiste en costillas espirales separadas por surcos, que cubren los dos tercios distales de la vuelta corporal; el color es blanquecino y está moteado por manchas pardo oscuras rectangulares espirales grandes y pequeñas, una hilera de las manchas grandes se localiza en los hombros carinados de la vuelta corporal y también permanece en las vueltas de la espira por encima de la sutura; abertura con seno posterior somero y canal anterior algo levantado hacia la izquierda, el interior es blanquecino y el labio externo trasluce las manchas externas. Longitud 25,5 mm; diámetro $11,0 \mathrm{~mm}$.

Distribución: Islas Cedros, Baja California, a través del Golfo de California y sur de Ecuador (Keen 1971), y Bocapán, Tumbes, Perú (Rivadeneira \& Injoque 1990).

Hábitat: Sublitoral en fondo areno fangoso a $24 \mathrm{~m}$ de profundidad (Rivadeneira \& Injoque 1990). Según Mulliner (1996), entre 50 y $60 \mathrm{~m}$ de profundidad.

Localidades: Tumbes (Las Salinas), Piura (La Bocana del Estero).

Material examinado: 2 lotes, 2 ejemplares; fondo fango arcilloso y areno fangoso, 24 - $30 \mathrm{~m}$ de profundidad.

\section{Conus (Ximeniconus) ximenes Gray, 1839}

\section{Figura 11}

Conus ximenes Gray, 1839. Zool. Beechey's Voyage : p. 119, pl. 3, fig. 2; Dall, 1909: 207; Abbott, 1974: 258, pl. 15, fig. 2823; Kerstitch, 1989: 53, fig.113; Hendrickx \& Toledano, 1994: 59. Conus (Chelyconus) ximenes, Peña, 1970: 169.

Conus (Ximeniconus) ximenes, Keen, 1971: 669, fig. 1517; Alamo \& Valdivieso, 1987:82; Paredes et al., 1999: 29; Rivadeneira \& Arias, 1999: 88; Skoglund, 2002: 165.

Concha algo más baja que en $C$. mahogani, la espira de perfil ligeramente cóncavo, mide aproximadamente la cuarta parte de la longitud de la conchilla; vuelta corporal con hombros no carinados; abertura amplia sin seno posterior y con amplio canal anterior; vuelta corporal presenta siete surcos anchos espirales espaciados que nacen en el labio columelar y terminan en el borde del labio externo, el resto de la concha es liso, espira con una serie de manchas irregulares, y el color de fondo de la conchilla es blanco violáceo con algunas bandas irregulares de color pardo oscuro, y en toda la vuelta corporal se distinguen hileras espirales de manchas pequeńas cuadradas y rectangulares, una hilera de manchas cuadradas algo más grandes, en los hombros y también bajo la sutura la sutura; interior de la abertura color lavanda pálido. Longitud 54,8 $\mathrm{mm}$; diámetro $27,2 \mathrm{~mm}$.

Distribución: Golfo de California; Panamá (Keen, 1971): islas Galápagos y Provincia del Guayas, Ecuador (Finet 1985, Cruz 2007) a Sechura, Perú (Alamo \& Valdivieso 1987).

Hábitat: Infralitoral areno-fangoso (Alamo \& Valdivieso 1987). Hasta los 91,4 m de profundidad (Kerstitch 1989).

Localidades: Tumbes (Zarumilla, Bocapán), Piura (Sechura, Vichayo).

Material examinado: 4 lotes, 32 ejemplares; fondo arenoso, $10 \mathrm{~m}$ de profundidad.

\section{GÉnero Conus, S.L.}

\section{Conus kohni MacLean \& Nybakken, 1979}

Figura 12

Conus kohni MacLean \& Nybakken, 1979. The Veliger Vol. 22 (2): 135-144, figs. 24-29, text fig. 4. ; Skoglund, 2002: 165; Tucker, 2008: 7-10, figs. $1-3,2 a$, text fig. $f$.

Concha más esbelta y con la espira más alta y aguzada que C. xanthicus, perfil de la espira ligeramente cóncavo, superficie de las tres últimas vueltas suavemente cóncavos, sutura bajo los hombros marcadamente acanalada; vuelta corporal con lados casi rectos, ligeramente convexos bajo los hombros que son subcarinados; abertura más ancha que en C. xanthicus, canal posterior de mediana profundidad, el labio columelar deprimido en su mitad distal amplía la abertura, y el labio externo se refleja dorsalmente; la concha es lisa y sólo presenta estrías vestigiales en el tercio distal de la vuelta corporal, no hay estrías en las vueltas de la espira; el color general es amarillo anaranjado con dos bandas discontinuas blanquecinas irregulares y un círculo de pequeñas motas claras en los hombros de la vuelta corporal, las mismas que se amplían en las vueltas de la espira, el interior de la abertura es blanco; no se observan restos de periostraco. Longitud 42,6 mm; diámetro 19,7 mm.

Distribución: Isla Espíritu Santos, Golfo de California. Baja California sur, Mexico (Tucker 2008), Islas Galápagos, Ecuador (MacLean \& Nybakken 1979).

Hábitat: En una variedad de tipos de fondo, en profundidades entre 18 y $100 \mathrm{~m}$, conjuntamente con C. xanthicus (MacLlean \& Nybakken 1979).

Nueva localidad: Tumbes $\left(03^{\circ} 34,5385^{\prime} S-81^{\circ} 11,309^{\prime} \mathrm{W}\right)$.

Material examinado: Un lote, un ejemplar, fondo rocoso a $121 \mathrm{~m}$ de profundidad (LaBSIM). Colector: Edgardo Enríquez (Lab. de Bentos Marino, IMARPE). Fecha: 10.09.2008.

Observaciones: Conus kohni MacLean \& Nybakken, 1979, fue considerado sinónimo de C. xanthicus (Walls 1980), pero Tucker (1985) sostuvo que es una especie válida, y finalmente Tucker (2008) estableció claramente las diferencias entre las dos especies. El hallazgo de Conus kohni MacLean \& Nybakken, 1979, es un nuevo registro para la malacofauna marina del Perú.

\section{Conus xanthicus Dall, 1910}

Figura 13

Conus xanthicus Dall, 1910. Pro. U. S. Nat. Mus. Vol. 38 (1741): 225; Keen,1971: 667, fig. 1511, derecha (como sinónimo de C. fergusoni); Skoglund,2002: 165; Tucker, 2008: 7-10, figs. 5, 5a, 6 ; figs. de texto, B, C.

Concha sólida, con espira baja de perfil ligeramente cóncavo; lados de la vuelta corporal casi rectos, ligeramente convexos cerca de los hombros que son subcarinados; superficie de las tres últimas vueltas de la espira algo cóncavas, la sutura bajo los hombros poco hundida; abertura con seno anal de mediana profundidad y labio externo curvado, más abierto en su extremo anterior; escultura con suave estriación espiral en las vueltas de la espira, y con estrías en, aproximadamente, el tercio anterior de la vuelta corporal, las estrías rodean la vuelta desde el labio interno hasta el borde del labio externo, el resto de la concha 
es liso; coloración de la conchilla es anaranjada oscura con dos bandas amarillentas irregulares interrumpidas y un círculo de motas del mismo color bajo los hombros, el interior de la abertura es blanco, la espira presenta un color más oscuro que el de la vuelta corporal debido a la presencia de manchas axiales de color pardo oscuro; periostraco pardo aterciopelado, más grueso en la espira. Longitud 39,4 mm; diámetro 19,4 mm.

Distribución: Morro Colorado, Sonora, México, hasta Colombia; islas Revillagigedo, México; islas Galápagos, Ecuador; incluye isla Danzante, Golfo de California, México (Skoglund 2002).

Hábitat: Infralitoral rocoso hasta $162 \mathrm{~m}$ de profundidad (Mulliner 1996).

Nueva localidad: Tumbes $\left(03^{\circ} 34,5385^{\prime} S-81^{\circ} 11,309^{\prime} \mathrm{W}\right)$.

Material examinado: Un lote, Un ejemplar; fondo rocoso, a $121 \mathrm{~m}$ de profundidad. (LaBSIM). Colector Edgardo Enríquez (Lab. de Bentos Marino, IMARPE). Fecha: 10.09.2008.

Observaciones: Dall (1910) describió la especie colectada frente a Guaymas, Mexico, a 71 brazas de profundidad en fondo arenoso. Conus chrysocestus Berry, 1968 es ahora sinónimo de Conus xanthicus Skoglund, 2002). Conus kohni MacLean \& Nybakken, 1979, también fue considerado sinónimo (Walls 1980), pero Tucker (1985) aclaró que es una especie válida. El hallazgo de Conus xanthicus Dall 1910, es un nuevo registro para la malacofauna marina del Perú.

\section{Agradecimientos}

Los autores agradecen al Instituto del Mar del Perú por haber propiciado la participación de Katherine Altamirano en la expedición a la costa norte del país el año 2007, dentro del Proyecto Caballito de Mar, actividad de la Unidad de Investigaciones en Biodiversidad, lo cual posibilitó la colecta de parte del material estudiado. Igualmente expresamos nuestro reconocimiento al Doctor Dimitri Gutiérrez Jefe del Laboratorio de Bentos Marino del Instituto del Mar del Perú, y al Blgo. Edgardo Enríquez, por habernos proporcionado los ejemplares de cónidos que fueron determinados como nuevos registros para el mar peruano. Asimismo, agradecemos al Consejo Superior de Investigaciones de la Universidad Nacional Mayor de San Marcos por el apoyo económico a los estudios sobre la diversidad de los moluscos marinos del Perú.

\section{Literatura citada}

Abbott R.T. 1974. American Seashells. Second edition. Van Nostrand Reinhold Company, New Cork, 663 pp. 24 pls.

Adams C.B. 1852. Catalogue of shells collected at Panama, with notes on their synonymy, station and geographical distribution. New York. 334 pp.

Alamo V. \& V. Valdivieso. 1987. Lista sistemática de moluscos marinos del Perú. Bol. Inst. Mar. Perú. Vol. extraordinario: 1-205.

Alamo V. \& V. Valdivieso. 1997. Lista sistemática de moluscos marinos del Perú. Bol. Inst. Mar. Perú. Vol. extraordinario: $1-183$.

Berry S. S. 1968. Notices of new eastern Pacific Mollusca- VII. Leaflets in Malacol., 1(25): 155-158.

Blanco J.F. \& J.R. Cantera. 1994. La familia Conidae (Mollusca: Gastropoda) en el Pacífico Colombiano. Boletín Ecotrópica 27: 19-39.
Broderip W.J. \& G.B. Sowerby. 1829. Observations on new or interesting Mollusca contained, for the most part, in the Museum of the Zoological Society. Zool. Jour., London, 4: 359- 379, pl. 9.

Broderip W.J. \& G.B. Sowerby. 1833. Genus Conus. Proc. of the Zoological Society of London. pp. 52-55.

Cantera J.M., E.A. Rubio, F.J. Borrero, et al. 1979. Taxonomía y distribución de los moluscos sublitorales de La Isla Gorgona, Colombia In H. von Prahl, M. Grogl, F. Guhl (Eds.). Gorgona. Universidad de los Andes, Bogotá. pp: 141-168

Chaney H.W. 1987. A comparative study of two similar Panamic cones: Conus ximenes and Conus mahogany. The Veliger 29(4): 428-436, figs. 1-6.

Cosel R. von. 1984. Moluscos marinos de la Isla de Gorgona (costa del Pacífico Colombiano). Anales del Instituto de Investigaciones Marinas de Punta Betín 14: 175-257.

Cruz M. 1992. Estado actual del recurso malacológico (Bivalvos y Gasterópodos) de la zona infralitoral del Golfo de Guayaquil. Acta Oceanográfica del Pacífico 7(1): 41-68.

Cruz M. 2007. Poliquetos y moluscos macrobentónicos de la zona intermareal y submareal de la Provincia del Guayas, Monteverde, Ecuador. Acta Oceanográfica del Pacífico 14(1): 147-153.

Dance S.P., \& R. von Cosel. 1977. Das grosse Buch der Meeresmuschelm: Schenecken und Muscheln der Weltmeere. Verlag E. Ulmer, Stuttgart, Germany, 304 pp.

Dall W.H. 1909. Report on a collection of shells from Peru, with a summary of the littoral marine mollusca of the Peruvian Zoological province. Proc. U.S.N. Mus. 37 (1704): 147294.

Dall W.H. 1910. Summary of the shells of the genus Conus from the Pacific coast of America in the United States National Museum. Proceedings of the United States National Museum 38(1741):217-228.

Díaz J., G. Adriana \& J. Cantera. 2005. Checklist of the Cone Shells (Mollusca: Gastropoda: Neogastropoda: Conidae) of Colombia. Biota Colombiana 6(1): 73- 86.

Gonzalez-Villarreal L.M. 2005. Guía ilustrada de los gasterópodos marinos de la Bahía de Tenacatita, Jalisco, México. Scientia-CUCBA 7(1):1-84.

Finet Y. 1985. Preliminary faunal listo of the marine mollusks of the Galápagos Islands. Institut Royal des Sciences Naturelles de Belgique. Documents de Travail 20: $50 \mathrm{pp}$.

Gray J.E. 1839. Molluscous animals and their shells. In F. W. Beechey, the Zoology of Captain Beechey's Voyage: 101-155.

Hanna G.D. 1963. West American Mollusks of the genus Conus-II. Ocasional papers of the California Academy of Sciences 35: 1-103, $11 \mathrm{pls}$.

Hendrickx M. \& A. Toledano. 1994. Catálogo de Moluscos Pelecypodos, Gasterópodos y Poliplacóforos. Catálogo de Referencia, Estación Mazatlán, ICML; UNAM. Inst. Cien. del Mar y Limnología, UNAM. 71 pp.

Hinds R.B. 1843. Descriptions of new shells from the collection of Captain Sir Edward Belcher. The Annals and Magazine of Natural History 11: 255-257.

Hutsell K., L. Hutsell \& D. Pisor. 2001. Hutsell and Pisor's Registry of World Record Size Shells. Third edition, 158 pp. Snail's Pace Productions, San Diego, California.

Keen A.M. 1971. Sea shells of tropical west America. 2th ed. Stanford Univ. Press, California. 1064 pp.

Keen A.M. \& E.V. Coan. 1975. Sea Shells of Tropical West America: additions and corrections to 1975 . The Western Society of Malacologists Occasional Paper 1: 66 pp.

Kerstitch A. 1989. Sea of Cortez Marine Invertebrates. Sea Challengers-Monterey, California. 114 pp., 283 figs. 
Kohn A.J. 1990. Tempo and Mode of Evolution in Conidae. Malacología 32(1): 55-67.

López J. 2001. Conotoxinas. Spira 1: 7-11.

McLean J.H. \& J. Nybakken. 1979. On the growth stages of Conus fergusoni Sowerby, 1873, the reinstatement of Conus xanthicus Dall, 1910, and a new species of Conus from the Galápagos Islands. The Veliger 22(2): 135-144, figs. $1-4,3$ pls.

Mogollón V. \& J. Vargas. 1999. Registro de Conus Asprella) arcuatus (Mollusca, Gastropoda, Conidae) en aguas peruanas. Revista Científica Wiñay Yachay 3(1): 21-26

Mulliner M. 1996. Dredging around Isla Danzante, Gulf of California, Mexico or what you find at the end of a line. The Festivus 28(6): 62-70, figs. 1-17.

Nybakken J. 1967. Preliminary observations on feeding behaviour of Conus purpurascens Broderip, 1833. Veliger 10(1): 55-57.

Nybakken J. 1970. Radular anatomy and systematics of the West American Conidae (Mollusca, Gastropoda). American Museum Novitates 2414: 1-29, figs. 1-45.

Nybakken J. 1988. Possible ontogenetic change in the radula of Conus patricius of the eastern Pacific. The Veliger 31(3/4): 222-225, figs. 1-2.

Nybakken J. 1990. Ontogenetic change in the Conus radula types, and significance in systematic and ecology. Malacologia 32(1): 35-54, figs. 1-13.

Paredes C., P. Huaman, F. Cardoso, R. Vivar \& V. Vera. 1999. Estado actual del conocimiento de los moluscos acuáticos en el Perú. Rev. peru. biol. 6(1): 5-47.

Peña G.M. 1970. Zonas de distribución de los gasterópodos marinos del Perú. An. Cient. Univ. Nac. Agraria 8 (3/4): 153-170.

Ramírez R., C. Paredes \& J. Arenas. 2003. Moluscos del Perú. Rev. Biol. Trop. 51(Suppl. 3): 225-284.

Reeve L.A. 1843. Monograph of the genus Conus. Conchologia Iconica, Í: figures and descriptions of the Shells of Molluscs; with remarks on their affinities, synonymy, and geographical distribution, 1. Conus.

Rivadeneira V. \& F. Injoque. 1990. Nuevo registro de caracol marino para el Perú. Boletín de Lima 70: 35-36, 1 fig.

Rivadeneira V. \& C. Arias. 1999. Especies peruanas del subgenero Ximeniconus Emerson \& Old Jr., 1962 (Mollusca, Gastropoda, Conidae). Boletín de Lima 118, pp. 87-93.
Röckel D., W. Korn \& A. Kohn. 1995. Manual of the living Conidae. Vol 1. Verlag Hemmen, Wiesbaden.

Rosenberg G. 1992. The Encyclopedia of Seashells. Michael Friedman Publishing Group, Inc., New York. 224 pp.

Shasky D.R. 1975. Range extensions for two tropical west American gastropds. The Veliger 18(2): 217.

Shasky D.R. 1984. A preliminary checklist of marine mollusks from Manabí Province, Ecuador. Annual Report of the Western Society of Malacologists 16: 25-32.

Shasky D.R. 1996. Distributional records of interesting and rarely collected marine gastropods from the Tropical Eastern Pacific. The Festivus 28(4): 35-45.

Shasky D. R. 1997. New range, depth and size for some Panmic Province gastropods. The Festivus 29(6):45-52, figs. 1-13.

Skoglund C. 2002. Panamic Province Molluscan literatura. Additions and changes from 1971 through 2001. III Gastropoda. The Festivus 33: 1-286.

Sowerby G.B.Jr. 1833. The Conchological Illustrations, pt. 29, fig. 29.

Sowerby G.B.Jr. 1873. Descriptions of five new cones. Proc. Zool. Soc. London, pp. 145-146, pl. 15.

Tomlin J.R.B. 1937. Catalogue of Recent and fossil cones. Proc. of the Malacological Society of London 22: 205-330.

Tucker J.K. 1985. Conus ximenes Gray, 1839, Conus mahogani (sic) Reeve, 1843 and friends. Shells and Sea Life 17(6): 201-202.

Tucker J.K. 2004. Catalog of the Coninae (version of 01/01/2004), Brighton, Illinois, USA.

Tucker J.K. 2007. Conus ximenes and Conus mahogany: two similar but distinct species. The Cone Collector 2: 5-10, pl. 1, figs. 1-12, pl. 2, figs. 1-7.

Tucker J.K. 2008. Distribution of Conus kohni McLean \& Nybakken, 1979. The Cone Collector 5: 7-10, figs. 1-11.

Walls J.G. 1979. Cone Shells a Synopsis of the Living Conidae. T. F. H. Publications, Neptune City, NewJersey, 1011 pp.

Walls J.G. 1980. Conus update: Sept. 1979 - Feb. 1980. Pariah 8: 3-6 (Sept. 30).

Wood W. 1828. Supplement to the Index testaceologicus; or a catalogue of shells, British and foreing. Ilustrated with 480 fig. London, 59 pp., 8 pls. 


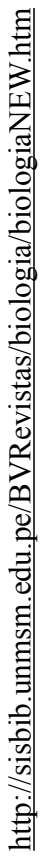

